\title{
Perceptions and Efficacy of Oral Rinsing with Two Types of Coconut Oil: A Comparative Study
}

\author{
Faizal C. Peedikayil ${ }^{*}$ Neethu P. Diwaker, Chandru T. P., Soni Kottayi
}

\begin{abstract}
This study focuses on the efficacy of virgin and regular coconut oil on plaque-related gingivitis and the perceptions of the subjects regarding its taste and odor. A study was carried out on 80 subjects, divided into 4 groups, 20 participants each. Group A: virgin coconut oil (VCO) gargling, Group B: regular cooking coconut oil (RCCO) gargling, Group C: chlorhexidine mouthwash gargling, and Group D: routine toothbrushing. The Modified gingival Index (MGI) was assessed to check the gingival inflammation on the $15^{\text {th }}$ and $30^{\text {th }}$ days. Perceptions of the subjects on the taste and odor were measured with the Hedonic Scale, and texture of VCO and RCCO in comparison with chlorhexidine. The baseline means MGI values are: $1.62 \pm 0.47,1.74 \pm 0.22,1.78 \pm 0.22,1.68 \pm 0.66$ for Group A, B, C, and D respectively. There is a significant difference in gingival index scores across all the study groups on the $15^{\text {th }}$ day and $30^{\text {th }}$ day (intra-group comparison). There is a significant difference in mean scores when group VCO, RCCO, and Chlorhexidine are compared with the control (inter-group comparison). Hedonic rating scale shows: chlorhexidine has a better odor (3.2) than VCO (3.1), RCCO (2.9). Chlorhexidine scored (3.4) in taste compared with VCO (3.1) and RCCO (2.8). Texture and mouthfeel scores for Chlorhexidine and VCO (3.6) and RCCO (3.4). VCO and RCCO are as efficient in reducing gingivitis. VCO has better taste, odor, and texture in the mouth than RCCO.
\end{abstract}

Key words: Efficacy, oral rinsing, virgin coconut oil, regular cooking coconut oil, mouthwash gargling

\section{INTRODUCTION}

Oil rinsing is a type of traditional procedure in the Indian system of medicine that involves swishing edible oil in the mouth and then spitting it out. This procedure is also called 'Oil Pulling' because the oil used for swishing is pulled and swirled to all parts of the oral cavity by movements of the tongue and oral musculature (Ripari et al., 2020). Ancient Ayurveda textbooks like Charaka Samhita and Arthashastra have mentioned these procedures as Kavala Gandoosha and Kavala Graha. Kavala Gandoosha is a procedure in which the mouth is completely filled with a large amount of oil and is spitted after a few minutes, whereas Kavala Graha is a procedure in which the oil is retained in the mouth and swished. Some Ayurveda textbooks say that such practices cure about 30 systemic diseases and have an effect on the overall well-being of the individuals practicing it (Pedikayil et al., 2015; Singla et al., 2015).

Oil rinsing or oil pulling is advised to be done in the morning on empty stomach, the oil is taken in the mouth before or after tooth brushing and is moved between the teeth for a few minutes till the

Department of Pediatric Dentistry, Kannur Dental College, India.

${ }^{*}$ Corresponding Author. Email: drfaizalcp@gmail.com 
oil turns thin and milky white and is spitted out (Peedikayil, 2019).

A variety of common edible oils are used for oil pulling therapy such as sesame oil, coconut oil, sunflower oil, groundnut oil, olive oil, mustard oil, and leaf extracts of gooseberries and mango. The advantage of these natural oils is that they neither cause any staining as seen in the use of mouthwashes nor there is any after taste or allergic reactions and are readily available (Shanbhag, 2016).

Various types of coconut oils are available in the market depending upon the method of extraction of oil from the coconut (Cocos nucifera L). In the present study, two types of coconut oil are considered for oil gargling. Regular Cooking Coconut Oil (RCCO) is made from dried coconut kernel called 'copra'. The copra is pressed; extracted oil is refined, decolorized and bleached. This process makes it suitable for consumption and has a high content of mediumchain fatty acids. Virgin coconut oil (VCO) is obtained from the fresh and mature kernel of the coconut by mechanical or natural means with or without the application of heat, which does not lead to alteration of the nature of the oil. VCO doesn't undergo any chemical refining, bleaching or deodorizing (Wallace, 2019; Deen et al., 2021; Dayrit et al., 2011).

Therefore, a study was conducted to compare the efficiency of regular coconut oil and virgin coconut oil in comparison with chlorhexidine mouth wash. The study also accesses the individual perceptions such as taste, odor and mouthfeel on its use.

\section{MATERIALS AND METHODS}

A prospective interventional comparative study was carried out in 80 male subjects in the age group of 14-18 years. The number of participants was calculated using the online software (https:// clincalc.com) Minimum number needed in each group was 18 , which was rounded to 20 in each group. Before the start of the study, ethical approval was obtained from the Institutional Review Board Committee (KDC/ETH/18/PED11/4A).

The inclusion criteria for the study were subjects with plaque-related gingivitis. Individuals with systemic disease, individuals on antibiotic or steroid medications and history of any dental treatment in the past 6 months, were excluded from the study. The study was explained to the participants and parents and informed written consent was obtained from the parents before proceeding with the study.

The selected study population was divided into four groups which included:

- Group A with a total of 20 participants for virgin coconut oil (VCO) gargling

- Group B with a total of 20 refined for regular cooking coconut oil (RCCO) gargling

- Group C with a total of 20 for chlorhexidine mouthwash gargling

- Group D (Control) with a total of 20 participants for routine toothbrushing only

The subjects designated for group A and group B were advised to routinely perform swishing for 3-4 minutes in the morning with $5 \mathrm{ml}$ of oil provided to them and group $\mathrm{C}$ participants were advised to routinely perform $5 \mathrm{ml}$ mouthwash gargling for 3-4 minutes in the morning. In addition, the subjects were directed to perform their routine tooth brushing 30 minutes after the oil/mouth wash gargling. While swishing, the subjects were advised to swish the fluids in all parts of the oral cavity. The participants in group D were advised to carry only routine tooth brushing only. All subjects were provided with a new toothbrush and toothpaste to use during the period of study and standard toothbrushing was demonstrated to them to ensure standard oral hygiene 
practices. The Modified gingival Index (MGI) was assessed at baseline $15^{\text {th }}$ day and $30^{\text {th }}$ day for all participants. Following criteria are adopted $0=$ absence of inflammation; 1 = mild inflammation or with slight changes in color and texture but not in all portions of gingival marginal or papillary gingiva; 2 = mild inflammation, in all portions of gingival marginal or papillary; 3 = moderate, bright surface inflammation, erythema, edema and/or hypertrophy of gingival marginal or papillary; 4 = severe inflammation: erythema, edema and/or marginal gingival hypertrophy of the unit or spontaneous bleeding, papillary, congestion or ulceration.

After 30 days of a 5-point questionnaire was given to the participants of group 1 and group 2 and group 3 to know their perceptions of using coconut oil swishing in comparison with coconut oil mouth wash use. A hedonic scale was used to find the acceptance of taste, odor and mouth feel of coconut oil.

The data obtained in the study were tabulated and analyzed using SPSS software (version 21, IBM, USA). One-way ANOVA was done to find the significance $(\mathrm{P}<0.5)$. Bonferroni post hoc test was done to compare mean scores within groups.

\section{RESULTS}

The baseline means MGI values obtained for each group are $1.62 \pm 0.47,1.74 \pm 0.22,1.78 \pm 0.22$, $1.68 \pm 0.66$ for group A, group B, group C, Group D respectively. Figure 1 shows the modified gingival index values obtained for rach groups at baseline, $15^{\text {th }}$ day and $30^{\text {th }}$ day. Table 1 shows an inter-group Comparison of mean modified gingival index scores at baseline, on the $15^{\text {th }}$ day and $30^{\text {th }}$ day between the groups. The results show that there is a significant difference across the study groups

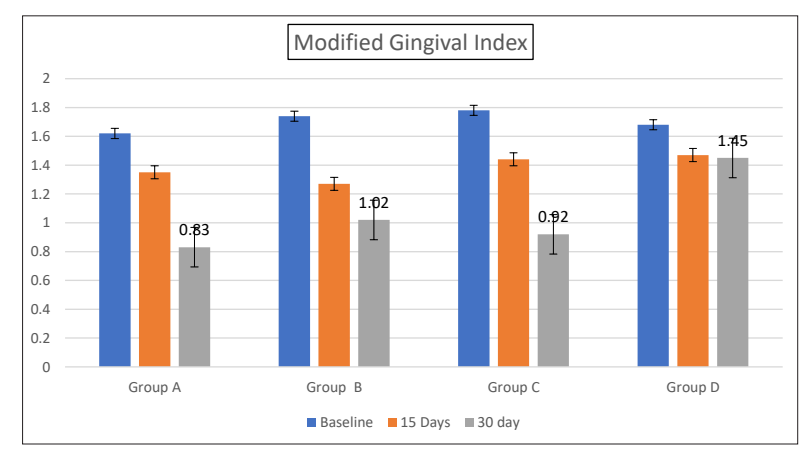

Figure 1. Modified Gingival Index at baseline, 15 and 30 days

\begin{tabular}{lcccccc}
\hline Time Period & $\begin{array}{c}\text { Group A } \\
\text { (mean with } \\
\text { SD) }\end{array}$ & $\begin{array}{c}\text { Group B } \\
\text { (mean with } \\
\text { SD) }\end{array}$ & $\begin{array}{c}\text { Group C } \\
\text { (mean with } \\
\text { SD) }\end{array}$ & $\begin{array}{c}\text { Group D } \\
\text { (mean with } \\
\text { SD) }\end{array}$ & F Value & Significance \\
\hline Baseline & $1.62 \pm 0.47$ & $1.74 \pm 0.22$ & $1.78 \pm 0.22$ & $1.68 \pm 0.66$ & 2.232 & 0.254 \\
$15^{\text {th }}$ day & $1.35 \pm 0.28$ & $1.27 \pm 0.26$ & $1.44 \pm 0.86$ & $1.47 \pm 0.30$ & 16.17 & $0.000 \#$ \\
$30^{\text {th }}$ day & $0.83 \pm 0.22$ & $1.02 \pm 0.28$ & $0.92 \pm 0.28$ & $1.55 \pm 0.32$ & 39.26 & $0.000 \#$ \\
\hline
\end{tabular}

One way ANOVA, \# $P<0.05$ = statistically significant

Table 1. Inter Comparison of mean modified gingival index scores

\begin{tabular}{lccccc}
\hline Time Period & Baseline & $15^{\text {th }}$ Day & $30^{\text {th }}$ Day & F Value & Significance \\
\hline Group A & $1.62 \pm 0.47$ & $1.35 \pm 0.28$ & $0.83 \pm 0.22$ & 51.5 & $0.02 \#$ \\
Group B & $1.74 \pm 0.22$ & $1.27 \pm 0.26$ & $1.02 \pm 0.28$ & 33.56 & $0.02 \#$ \\
Group C & $1.78 \pm 0.22$ & $1.44 \pm 0.86$ & $0.92 \pm 0.28$ & 44.76 & $0.02 \#$ \\
Group D & $1.68 \pm 0.66$ & $1.47 \pm 0.30$ & $1.55 \pm 0.32$ & 29.98 & 0.452 NS \\
\hline
\end{tabular}

$P<0.05$ = statistically significant, $\#$ denotes significance

Table 2. Intra-group comparison of gingivitis scores at different time periods 


\begin{tabular}{lcccccc}
\hline Time Period & $\begin{array}{c}\text { Group A } \\
\text { vs Group B }\end{array}$ & $\begin{array}{c}\text { Group A } \\
\text { vs Group C }\end{array}$ & $\begin{array}{c}\text { Group A } \\
\text { vs Group D }\end{array}$ & $\begin{array}{c}\text { Group B } \\
\text { vs Group C }\end{array}$ & $\begin{array}{c}\text { Group B } \\
\text { vs Group D }\end{array}$ & $\begin{array}{c}\text { Group C } \\
\text { vs Group D }\end{array}$ \\
\hline Baseline & 0.543 & 0.343 & 0.725 & 0.823 & 0.634 & 0.732 \\
$15^{\text {th }}$ day & 0.876 & 0.363 & $0.034 \#$ & 0.652 & $0.012 \#$ & $0.043 \#$ \\
$30^{\text {th }}$ day & 0.654 & 0.876 & $0.022 \#$ & 0.735 & $0.047 \#$ & $0.036 \#$ \\
\hline
\end{tabular}

Bonferroni post hoc test $(P<0.05=$ significant $)$. \# denotes significance

Table 3. Comparison of mean gingivitis scores between study groups using Bonferroni post hoc test

\begin{tabular}{|c|c|c|c|c|c|c|c|c|c|c|c|c|c|c|c|c|c|c|c|c|c|c|c|}
\hline & Ratings by & $\operatorname{art}$ & $\operatorname{cip}$ & ants & & & & & & & & & & & & & & & & & & Total & Average \\
\hline \multirow{3}{*}{$\begin{array}{l}\text { Virgin } \\
\text { Coconut } \\
\text { Oil }\end{array}$} & Aroma & 4 & 3 & 3 & 3 & 3 & 4 & 3 & 3 & 3 & 2 & 3 & 3 & 3 & 3 & 3 & 2 & 3 & 4 & 4 & 3 & 62 & 3.1 \\
\hline & Taste & 4 & 3 & 3 & 4 & 4 & 3 & 3 & 3 & 3 & 2 & 3 & 2 & 3 & 3 & 3 & 3 & 3 & 3 & 3 & 3 & 62 & 3.1 \\
\hline & $\begin{array}{l}\text { Texture } \\
\text { Mouthfeel }\end{array}$ & 3 & 4 & 4 & 4 & 3 & 4 & 4 & 3 & 4 & 4 & 3 & 3 & 3 & 3 & 4 & 4 & 4 & 4 & 3 & 4 & 72 & 3.6 \\
\hline \multirow{3}{*}{$\begin{array}{l}\text { Regular } \\
\text { Cooking } \\
\text { Coconut } \\
\text { Oil }\end{array}$} & Aroma & 3 & 3 & 2 & 3 & 3 & 4 & 3 & 3 & 3 & 2 & 3 & 4 & 3 & 3 & 3 & 2 & 4 & 2 & 2 & 3 & 58 & 2.9 \\
\hline & Taste & 2 & 3 & 3 & 2 & 4 & 3 & 3 & 3 & 3 & 2 & 3 & 2 & 3 & 3 & 3 & 3 & 3 & 2 & 2 & 3 & 56 & 2.8 \\
\hline & $\begin{array}{l}\text { Texture } \\
\text { Mouthfeel }\end{array}$ & 3 & 2 & 3 & 4 & 5 & 3 & 4 & 3 & 4 & 4 & 4 & 3 & 4 & 3 & 3 & 4 & 4 & 3 & 3 & 2 & 68 & 3.4 \\
\hline \multirow{3}{*}{$\begin{array}{l}\text { Chlorhex } \\
\text { idine }\end{array}$} & Aroma & 4 & 3 & 4 & 4 & 3 & 2 & 3 & 3 & 3 & 3 & 3 & 3 & 3 & 3 & 3 & 3 & 3 & 4 & 4 & 3 & 64 & 3.2 \\
\hline & Taste & 4 & 3 & 4 & 3 & 3 & 4 & 4 & 3 & 3 & 2 & 3 & 2 & 3 & 4 & 5 & 3 & 4 & 3 & 4 & 4 & 68 & 3.4 \\
\hline & $\begin{array}{l}\text { Texture } \\
\text { Mouthfeel }\end{array}$ & 4 & 4 & 4 & 4 & 3 & 4 & 4 & 3 & 4 & 4 & 3 & 3 & 4 & 3 & 4 & 4 & 2 & 4 & 4 & 3 & 72 & 3.6 \\
\hline
\end{tabular}

Like a lot $=5$, like a little $=4$, neither like or dislike $=3$ dislike a little $=2$ dislike a lot $=1$

Table 4. Perceptions of subjects using Hedonic Rating scale

on the $15^{\text {th }}$ day and $30^{\text {th }}$ day. there is no statistical difference in mean gingival index scores in the control group (one-way ANOVA, $P<0.05$ ).

Table 2 shows the intragroup comparisons. statistically significant changes from baseline to 30 days in all groups whereas no statistical significance is seen in the control group.

Table 3 shows a comparison of mean gingivitis scores between study groups using the Bonferroni post hoc test. The results show as there is a highly statistical difference in mean scores when groups A, B and C are compared with control Group D, ie Group A vs Group $\mathrm{D}\left(\mathrm{p}=0.034\right.$ on $15^{\text {th }}$ day and $\mathrm{p}=0.022$ on $30^{\text {th }}$ day), Group B vs Group D ( $\mathrm{P}=0.012$ on $15^{\text {th }}$ day and $p=0.047$ on $30^{\text {th }}$ day), group $C$ vs group $D$ $\left(\mathrm{p}=0.043\right.$ on $15^{\text {th }}$ day $\mathrm{p}=0.36$ on $30^{\text {th }}$ day).
Table 4 shows the perceptions of the subjects using different mouthwashes/oils as a part of this study. Hedonic rating scale shows that chlorhexidine has a better odor (3.2) than VCO (3.1) followed by RCCO (2.9). Chlorhexidine scored higher (3.4) in taste when compared with VCO (3.1) and RCCO (2.8). Texture and mouthfeel scores were the same for Chlorhexidine and virgin coconut oil (3.6) followed by RCO (3.4).

\section{DISCUSSION}

Chemo mechanical procedures is a part of oral hygiene maintenance as it reduces the incidence of plaque-related diseases such as gingivitis by decreasing plaque accumulation (Peedikayil, 2015). Modified Gingival Index is used for clinical assessment as it is the most widely used indices in trials for therapeutic 
agents. The Modified Gingival Index (MGI) uses a visual scale to assess gingival health. The MGI relies on a visual assessment of gingival changes to measure the severity of inflammation (Asokan et al., 2009). The results of our study show that there was a significant decrease in the gingival index at the end of 15 days and 30 days on coconut oil rinsing especially with virgin coconut oil and is comparable to chlorhexidine which is considered as a gold standard among antiplaque and gingivitis agents.

Oil rinsing helps in decreasing plaque accumulation thereby and gingival inflammation. The mechanical shear forces exerted on the oil during swishing leads to an increase in the surface area of the oil film. The oil film thus formed on the surface of the teeth can reduce plaque adhesion and bacterial co-aggregation. It was also proposed that the alkalis in the saliva can react with the oil leading to saponification and formation of a soaplike substance which can reduce the adhesion of plaque. Coconut oil has a high saponification value and is one of the most commonly used oils in making soaps. Coconut oil-based soaps can lather well and have an increased cleansing action. The lauric acid in the coconut oil can easily react with sodium hydroxide in saliva during oil pulling to form sodium laureate, the main constituent of soap which might be responsible for the cleansing action and decreased plaque accumulation (Peedikayil et al., 2015; Singla et al., 2014; Peedikayil et al., 2016). Another reason for the action of the coconut oil in the oral cavity may be that the lipase enzyme present in the saliva is responsible for the breakdown of Medium Chain fatty acids and therefore lauric acid can enhance the anti-inflammatory effect in the oral cavity (Lai, 2019).

Virgin coconut oil (VCO) consists mainly of medium-chain triglycerides, which are resistant to peroxidation. The fatty acids in virgin coconut oil are distinct from animal fats which contain mainly long-chain saturated fatty acids. Virgin coconut oil is colorless, free of sediment with a natural fresh coconut scent. It is free from rancid odor or taste whereas refined coconut oil is refined by neutralization with alkali, bleached with bleaching earth or activated carbon or both and deodorized with steam; no other chemical agents being used. One of the most immediate differences between Virgin and regular cooking coconut oil is the taste and aroma. While Virgin Coconut Oil boasts a delicious, tropical coconut scent and flavor, Regular cooking Coconut Oil has a mild coconut scent and flavor (Deen et al., 2021; Dayrit et al., 2011).

Polyphenols are abundant dietary micronutrients protecting cells from damage due to oxidative stress. Several phenols have been identified in coconut oil such as protocatechuic acid, vanillic, caffeic, ferulic, and p-coumaric acids (Dimzon et al., 2011). Marina et al. in Williamson (2017) found phenolic content was $7 \%$ higher in Virgin coconut oil than in refined coconut oil. Polyphenol amount was highest in virgin coconut oil produced by fermentation and lowest in refined coconut oil. This may also be the reason for better action of virgin coconut oil than regular cooking coconut oil in our study.

The present study also shows that coconut oil pulling and chlorhexidine gargling when used as an adjuvant has a statistically significant reduction in modified gingival index scores when compared to routine oral hygiene maintained with brushing alone. Coconut oil contains a high amount of lauric acid and has been shown to reduce markers of inflammation in animal studies (Wallace, 2019). Peedikayil et al. (2015) in a study found that the coconut oil pulling practice reduces plaque formation and plaque-induced gingivitis significantly from day 7 of oil pulling, and the scores showed a continued decrease during the study period of 30 days. A study by Kaliamoorthy et al. (2018)showed coconut oil gargling showed a significant reduction in the severity of gingivitis in the coconut oil group than the sesame oil 
group at all postintervention stages of their study. In the latest study by Ripari et al. (2020), coconut oil pulling showed a significant decrease in reducing plaque formation and gingivitis. The results of these studies are in agreement with our study results. In another study by Sezgin (2019) to find the plaque-inhibiting effects of oil pulling using 4-days plaque regrowth study model compared to $0.2 \%$ chlorhexidine gluconate (CHX) containing mouth rinse concluded that coconut therapy presented similar inhibitory activity on plaque regrowth compared with chlorhexidine.

Coconut oil is a readily accessible and cheap material for most when compared to chlorhexidine. Chlorhexidine mouthwashes are the most effective chemotherapeutic agent and are considered a gold standard against plaque-related gingivitis. But some studies have shown that Chlorhexidine on long-term use may alter taste sensation and also induce staining on the teeth surfaces. The mucous membranes and the tongue can also be affected and may be related to the precipitation of chromogenic bacteria (James et al., 2017).

The study also took into account the perceptions of the subjects regarding the Characteristic such as aroma, taste, texture/ mouthfeel of the oils/ mouth wash by using the Hedonic Rating Scale. Hedonic Rating scale is a widely used scale for measuring the acceptability of foods and beverages (Pimentel et al., 2016). Results show that chlorhexidine has a better taste and aroma. Among the oils tested virgin coconut oil has a better aroma, taste and texture taste of the oil. The perceptions of taste vary from person to person. Virgin coconut oils have a natural coconut flavor whereas regular cooking oil has a delicate, nutty flavor without a strong coconut taste. Future studies can be directed towards improving the aroma, taste and texture/ mouthfeel by the addition of certain herbs or natural substances without compromising on the efficacy of the coconut oil used as a mouth wash.
The limitations of this study are that the study is of short duration. The perceptions reported by the patients are based on a limited number of participants and can vary from person to person. Therefore, future studies have to be based on a greater number of participants and longer time period to check for long term efficacy and side effects.

\section{CONCLUSION}

This study focuses on the effectiveness of two different types of coconut oil in the rinsing procedure. The study is of clinical merit and proves that coconut oil is efficient, safe natural adjuvant to routine oral hygiene procedures. Virgin coconut oil and Regular cooking coconut oil safely used for oral swishing and can be an adjuvant to routine oral hygiene procedures. Taste perceptions need to be improved for better compliance with the oil rinsing procedure. More research has to be carried out to find a suitable natural flavoring addictive for better aroma, taste and oral texture of coconut oil to be used as a mouth wash.

\section{REFERENCES}

Asokan, S., Emmadi, P., \& Chamundeswari, R. (2009). Effect of oil pulling on plaque induced gingivitis: A randomized, controlled, triple-blind study. Indian Journal of Dental Research, 20(1), 47.

Dayrit, F. M., Dimzon, I. K. D., Valde, M. F., Santos, J. E. R., Garrovillas, M. J. M., \& Villarino, B. J. (2011). Quality characteristics of virgin coconut oil: comparisons with refined coconut oil. Pure and Applied Chemistry, 83(9), 1789-1799.

Deen, A., Visvanathan, R., Wickramarachchi, D., Marikkar, N., Nammi, S., Jayawardana, B. C., \& Liyanage, R. (2021). Chemical composition and health benefits of coconut 
oil: an overview. Journal of the Science of Food and Agriculture, 101(6), 2182-2193.

Dimzon, I. K. D., Valde, M. F., Santos, J. E. R., Garrovillas, M. J., Dejarme, H. M., Remollo, J. M. W., \& Dayrit, F. M. (2011). Physicochemical and microbiological parameters in the deterioration of virgin coconut oil.

James, P., Worthington, H. V., Parnell, C., Harding, M., Lamont, T., Cheung, A., ... \& Riley, P. (2017). Chlorhexidine mouthrinse as an adjunctive treatment for gingival health. Cochrane Database of Systematic Reviews, (3).

Kaliamoorthy, S., Pazhani, A., Nagarajan, M., Meyyappan, A., Rayar, S., \& Mathivanan, S. (2018). Comparing the effect of coconut oil pulling practice with oil pulling using sesame oil in plaque-induced gingivitis: A prospective comparative interventional study. Journal of Natural Science, Biology and Medicine, 9(2), 165.

Lai, W. Y. W., Chua, J. W. M., Gill, S., \& Brownlee, I. A. (2019). Analysis of the lipolytic activity of whole-saliva and site-specific secretions from the oral cavity of healthy adults. Nutrients, 11(1), 191.

Peedikayil, F. C. (2019). Is coconut oil good for oral health? A review. Journal of Health Research and Reviews, 6(1), 1.

Peedikayil, F. C., Remy, V., John, S., Chandru, T. P., Sreenivasan, P., \& Bijapur, G. A. (2016). Comparison of antibacterial efficacy of coconut oil and chlorhexidine on Streptococcus mutans: An in vivo study. Journal of International Society of Preventive \& Community Dentistry, 6(5), 447.

Peedikayil, F. C., Sreenivasan, P., \& Narayanan, A. (2015). Effect of coconut oil in plaque related gingivitis-A preliminary report. Nigerian medical journal: journal of the Nigeria Medical Association, 56(2), 143.

Pimentel, T. C., da Cruz, A. G., \& Deliza, R. (2016). Sensory evaluation: sensory rating and scoring methods.

Ripari, F., Filippone, F., Zumbo, G., Covello, F., Zara, F., \& Vozza, I. (2020). The Role of Coconut Oil in Treating Patients Affected by PlaqueInduced Gingivitis: A Pilot Study. European Journal of Dentistry, 14(04), 558-565.

Sezgin, Y., Ozgul, B. M., \& Alptekin, N. O. (2019). Efficacy of oil pulling therapy with coconut oil on four-day supragingival plaque growth: A randomized crossover clinical trial. Complementary therapies in medicine, 47, 102193.

Shanbhag, V. K. L. (2017). Oil pulling for maintaining oral hygiene-A review. Journal of traditional and complementary medicine, 7(1), 106-109.

Singla, N., Shashidhar Acharya, S. M., \& Singla, R. (2014). Effect of oil gum massage therapy on common pathogenic oral microorganisms-A randomized controlled trial. Journal of Indian Society of Periodontology, 18(4), 441.

Wallace, T. C. (2019). Health effects of coconut oil-A narrative review of current evidence. Journal of the american college of nutrition, 38(2), 97-107.

Williamson, G. (2017). The role of polyphenols in modern nutrition. Nutrition bulletin, 42(3), 226-235. 